\title{
L’analyse De L'impact Des Dimensions Éthiques Et Culturelles Sur L'intégration Relationnelle Des Supply Chains : Cas Du Maroc
}

\author{
Akhlaffou Mohamed \\ Lechheb Hafsa \\ Ecole Nationale de Commerce et de Gestion, \\ Université Ibn Zohr, Agadir, Maroc
}

doi: 10.19044/esj.2016.v12n13p118 URL:http://dx.doi.org/10.19044/esj.2016.v12n13p118

\begin{abstract}
This article aims to explore the determinants of Moroccan culture and analyze their influence on the relational integration of Supply Chains, by answering the following research question: Does Moroccan culture present favorable characteristics to the relational integration of Supply Chains? Given the fact that the relational integration of Supply Chains (Bowersox et al., 1996, 1999,2000) represents the cornerstone of the philosophy of Supply Chain Management (Mentzer et al., 2001). And since the national culture showed its influence on values and lifestyles (Sun, Horn and Merritt, 2004), on negotiations (Adair and Brett, 2005), and on behavior and ethics (Elahee, Kirby and Nasif, 2002). Consequently, we can estimate that national culture can have influence on the integration of Supply Chains and the building of trust between partners. The methodology adopted is both qualitative and quantitative, and is inscribed in the framework of moderate positivist epistemological position. The combination of methods will lead us to identify and deeply understand our research problematic.
\end{abstract}

Keywords: Supply Chain Management, National Culture, Integration, Trust, Moroccan Companies

\section{Résumé}

Le présent article a pour but d'explorer les caractéristiques de la culture marocaine, et d'analyser leur influence sur l'intégration relationnelle des Supply Chains, et cela en répondant à la question de recherche suivante : la culture marocaine présente-elle des caractéristiques favorables à l'intégration relationnelle des Supply Chains ? Etant donné que l'intégration relationnelle des Supply Chains (Bowersox et al, 1996, 1999, 2000) représente la pierre angulaire de la philosophie du Supply Chain 
Management (Mentzer et al, 2001). Et puisque la culture nationale a démontré son influence sur les valeurs et les modes de vie (Sun, Horn et Merritt, 2004), sur les négociations (Adair et Brett, 2005), sur les comportements et l'éthique (Elahee, Kirby et Nasif, 2002). Par conséquent, nous pouvons estimer que la culture nationale peut avoir des effets sur l'intégration relationnelle des Supply Chains, et sur le fondement de la confiance entre les partenaires. La méthodologie adoptée est à la fois qualitative et quantitative, qui s’inscrit dans le cadre du positionnement épistémologique positiviste aménagé. La combinaison des méthodes va nous conduire à cerner et à appréhender profondément notre problématique de recherche.

Mots clés : Supply Chain Management, Culture Nationale, Intégration, Confiance, Entreprises Marocaines.

\section{Introduction}

Une des tendances les plus remarquables sur laquelle il faut mettre l'accent par les entreprises, est la mondialisation. Dans le passé, beaucoup d'organisations étaient en compétition avec d'autres entreprises dans leur pays d’origine ou dans leur région. Aujourd'hui, les entreprises énumèrent de multiples partenaires partout dans le monde, s'approvisionnent en matières premières, demandent des prestations, développent des alliances et distribuent leurs produits dans tous les continents.

Désormais les entreprises sont sollicitées pour bâtir de puissantes relations avec leurs partenaires ; basées principalement sur la confiance, et à intégrer leurs Supply Chains, afin d'atteindre parfaitement les objectifs escomptés. Il y a un consensus (Barratt.M et Barratt.R, 2011) dans la littérature sur le fait que plus le niveau des relations avec les partenaires de la Supply Chain est élevé, plus la visibilité des activités opérationnelles des fournisseurs est grande. Cela va engendrer une certaine transparence et va contribuer à la création d'une plate-forme sur laquelle l'information peut être communiquée entre les acteurs. L’intégration des Supply Chains améliore le partage d'information, en engendrant des relations basées sur la confiance (Kim SW, 2009). En outre, l’approfondissement des liens basés sur la confiance entre les maillons de la Supply Chain, encourage la résolution des conflits et favorise la réactivité et la flexibilité entre tous les acteurs.

La confiance, en général, peut être définie comme la conviction profonde que l'associé avec qui l'échange a eu lieu est honnête et/ou bienveillant. La confiance, ainsi définie, est considérée comme un amortisseur saillant de la stabilité à long terme et du succès des relations inter organisationnelles (Flynn.BB, Huo.B, Zhao.X, 2010). 
La confiance comme l'appelle Arrow (1974), est le lubrifiant des relations sociales, qui en assure la durabilité. Les chercheurs s'appliquent à démontrer la fonction de la confiance dans la réduction des coûts de transaction (Zaheer et al., 1998 ; Dyer et Chu, 2003 ; Simon, 2007 ; Williamson, 1990), dans la stabilité des relations et la réduction des conflits (Marandon, 2003 ; Ring et Van de Ven, 1994) et dans la diminution des risques (Luhmann, 2006 ; Giddens, 1994, Delerue et Bérard, 2007). Certains parmi ces chercheurs, notamment Delerue et Bérard (2007), Dyer (1997), Charreaux(1997) et Thuderoz (1999), considèrent que la confiance est un mécanisme de coordination à part entière qui vient compléter les autres mécanismes de coordination identifiés par Williamson (1985). Finalement, la confiance ne peut que représenter un élément crucial, inévitable et antécédent de l'intégration des Supply Chains et de la durabilité des relations de leurs maillons.

Cependant, l'étude de l'intégration des Supply Chains, ainsi que la confiance ne peut être effectuée, sans la prise en compte d'un élément important responsable de leur succès ou de leur échec, celui de la culture nationale. En effet, beaucoup d'études récentes indiquent que les pratiques de gestion et leur efficacité, changent amplement d'un pays à l'autre. De telles variations sont inhérentes à la multiplicité des valeurs et des croyances culturelles (Ali, 1988, 1989; Ali and Azim, 1993; Hofstede, 1980; Hofstede and Bond, 1988; Ralston et al. 1993; Selmer and de Leon,1993).

Le but de cet article de recherche, est d'explorer les déterminants de la culture marocaine, et d'analyser comment ses caractéristiques qui peuvent favoriser l'intégration des Supply Chains, en nous focalisant, sur l'aspect relationnel de l'intégration des Supply Chains, et cela, en nous basant sur une variable médiatrice qui est la confiance.

\section{La culture nationale et l'intégration relationnelle des Supply Chains : fondements théoriques et débat}

Le Maroc illustre un pays qui a un potentiel énorme d'amélioration de la compétitivité de son économie, notamment au travers d'une logistique considérable, optimisant et ordonnant tous les flux de marchandises. De ce fait, le Maroc a mis en œuvre la Stratégie Nationale de Développement de la Compétitivité Logistique (2010-2015), qui va dégager des réponses cruciales pour le rayonnement de la logistique et qui ambitionne d'inscrire le Royaume du Maroc dans les Supply Chains les plus performantes.

La prise en compte de la culture nationale dans l'intégration des Supply Chains, demeure un point central à évoquer lors de l'adoption de ce nouveau mode de gestion, afin de contribuer à son succès.

Cet article de recherche a pour intérêt, d'explorer les déterminants de la culture marocaine et d'analyser leur impact sur l'intégration des Supply 
Chains. Cela en répondant à la problématique suivante: La culture marocaine présente-t-elle des caractéristiques favorables à l'intégration relationnelle des Supply Chains ?

Notre travail de recherche s'inscrit dans plusieurs champs théoriques, puisqu'il combine entre deux champs disciplinaires à savoir : l’intégration des Supply Chains et la culture nationale, et puisque son but est d'étudier l'influence de la culture nationale sur l'intégration des Supply Chains et sur la confiance entre les divers partenaires.

Les champs théoriques de notre article vont se présenter comme suit :

\section{Le Modèle de la culture nationale de Geert Hofstede}

Afin de traiter les déterminants de la culture nationale, nous avons opté pour le modèle de Geert Hofstede. Bien que divers modèles culturels sont proposés dans la littérature, et malgré beaucoup de critiques (Mc Sweeny, 2002), l'applicabilité du modèle d’Hofstede dans les travaux inscrits en sciences de gestion, et sa validité, demeurent remarquables, comparé à d'autres modèles culturels (Merrit, 2000, Magnusson et al, 2008). Le modèle d'Hofstede est adopté également dans les travaux qui ont pour objectif la comparaison entre les cultures nationales (Magnusson et al, 2008, Merrit, 2000). Finalement, le modèle d'Hofstede représente le modèle fondateur de la culture nationale, et montre une certaine facilité de compréhension, avec une explication claire des déterminants de la culture nationale.

La culture nationale peut être définie selon Hofstede (2010), comme une programmation mentale ; car cette programmation débute à l'intérieur de la famille, et se développe, ensuite, à l'école, dans le quartier, sur les lieux de travail et généralement dans la vie en groupe. L’anthropologie sociale montre que la culture nationale est l'addition des façons de penser, d'agir et de ressentir. La culture nationale, selon Hofstede, est donc, la programmation collective de l'esprit qui différencie les membres d'un groupe à des membres d'un autre groupe.

Le modèle de Geert Hofstede est composé de cinq principales dimensions de la culture nationale :

- $\quad$ La distance hiérarchique :

La distance hiérarchique est définie par Hofstede (1980), comme la perception du degré d’inégalité du pouvoir entre un subordonné et son supérieur. Ce déterminant fait référence au degré d’inégalité attendu et accepté par les individus. Dans les sociétés à forte distance hiérarchique, le pouvoir est centralisé et le leadership est exercé de manière autocratique et coercitive (Hofstede, 1983). Contrairement aux pays où la distance hiérarchique est faible, l’accent est mis sur l’égalité et sur la coopération. 
- $\quad$ L'orientation individualiste ou communautaire :

Selon Hofstede, la distinction entre les cultures nationales peut se faire également, à travers les relations que les individus entretiennent avec les autres membres de la collectivité.

Cette dimension renvoie à l'importance donnée par un individu à sa propre personne ou à son entourage (Hofstede, 1983, 1991). Dans les sociétés marquées par l'individualisme, les individus se préoccupent davantage de leurs propres intérêts et de leur environnement immédiat (Hofstede, 1994). Cependant, dans les pays à culture communautaire, l'importance est donnée aux liens affectifs et aux relations de confiance unissant les membres de la communauté.

- $\quad$ La relation à l'incertitude et à l'ambigüité :

Cette troisième dimension renvoie au degré de tolérance de l'incertitude et de l'ambigüité. Le niveau de contrôle de l'incertitude signifie; le degré jusqu'où les individus d'une culture acceptent les situations ambigües, risquées, imprévisibles, non-structurées, voire anarchiques. Aussi la manière avec laquelle ces membres réagissent à cette imprévisibilité (Hofstede, 1983,1991).

Le contrôle de l'incertitude peut être mis en relief à travers des plans et des outils de prévision, de recherche de la stabilité et de l'ordre. Le faible degré de contrôle de l'incertitude, se traduit par la prise de risque, et la confrontation à des événements futurs ambigus.

- $\quad$ L'orientation masculine ou féminine des valeurs :

Les cultures masculines accordent une grande importance aux valeurs de la réussite et de la possession, alors que les cultures féminines mettent l'accent surtout, sur les valeurs de l'entraide et de l'empathie, dans un souci de cohésion sociale.

- L'orientation à court terme et l'orientation à long terme :

Les cultures à orientation à court terme, sont des cultures qui respectent la tradition, l'exécution des obligations sociales, et considèrent que le service à autrui est un but important. Ces cultures ont comme valeurs principales du travail : la liberté, le respect des droits, la réussite et l'épanouissement personnel. Les cultures à orientation à long terme encouragent la persévérance, la prévoyance et le sens de l'épargne. Ces cultures ont comme fondamentales valeurs du travail: l'apprentissage, l'honnêteté, la responsabilité et l'autodiscipline. $\mathrm{Vu}$ que nous étudions les déterminants de la culture marocaine, et pour respecter et refléter au maximum la réalité de notre contexte de recherche, nous allons ajouter un autre déterminant même s'il ne figure pas dans le modèle d'Hofstede, celui de la religiosité.

En effet, bien que la religiosité ne figure pas parmi les déterminants de la culture nationale proposés par Hofstede, nous confortons notre choix 
par des travaux antérieurs (Hofstede, 1991, Schwartz et Huismans, 1995, Saroglou et al, 2004), qui ont intégré la religiosité, afin de mieux appréhender quelques sociétés caractérisées par la religion. Cette dernière peut, en effet, jouer le rôle d'un facteur explicatif des attitudes, des comportements et des relations avec autrui.

La société marocaine, est justement, en grande partie marquée par la religion (Balambo, 2012,2013) et un éventail de travaux montrent la religiosité de la société marocaine (Geertz, 1973, d’Iribarne, 1997, Al Maache, 2002, Bourquia et al, 2007, Tory et al, 2007).

Finalement, comme l’ont déjà confirmé, d’Iribarne (1997) et Geertz (1973), la culture marocaine est caractérisée par un fort attachement à la religion.

\section{La théorie des coûts de transaction (Oliver E. Williamson)}

Le sujet de l'intégration des Supply Chains peut être abordé à travers diverses théories, l'une de ces principales théories est la théorie des coûts de transaction, car cette théorie s'intéresse principalement aux sujets de collaboration, d'alliances et de relations inter-organisationnelles en général.

- $\quad$ Présentation générale de la théorie des coûts de transaction

La théorie des coûts de transaction suggère comme élément fondamental, que les marchés et les organisations sont des modes de coordination des échanges. C'est en comparant les coûts de transaction sur le marché avec ceux exigés par la hiérarchie, que la forme adéquate de gouvernance devient claire. Oliver E. Williamson est le chef de fil de l'économie des coûts de transaction. En addition à la proposition d'hypothèses de comportement à la base de cette théorie, cet auteur a réfléchi sur les formes de gouvernance les plus adéquats suivant les caractéristiques des transactions (Williamson, 1975, 1985). Il a aussi traité le sujet des formes intermédiaires de gouvernance, celles qui se situent à mi-chemin entre le marché et la hiérarchie (alliances, joint-ventures, franchises, intégration verticale à la japonaise). Et finalement, il étudie les types de contrats pour extraire une théorie générale du choix des formes de gouvernance. La théorie des coûts de transaction repose sur des hypothèses de comportement, qu'on affecte à un individu similaire à un homocontractor. Il s’agit de la rationalité limitée et de l’opportunisme.

La rationalité limitée est définie comme les limites des capacités cognitives des individus, qui permettent de prendre des décisions satisfaisantes plutôt que des décisions optimales. L’opportunisme est défini comme la recherche de l'intérêt personnel par le moyen de la tromperie, de la ruse, ou le plus souvent par le partage d'une information incomplète. La théorie des coûts de transaction présume qu'il existe des raisons économiques de faire un choix entre le marché ou la hiérarchie, comme des 
moyens de coordination des échanges. Ces raisons sont relatives aux caractéristiques des transactions, sur lesquelles il faut se baser pour choisir la forme de gouvernance la plus adéquate. Ces caractéristiques sont: la spécificité des actifs, l’incertitude et la fréquence.

La théorie des coûts de transaction est très pertinente pour traiter des sujets relatifs aux relations organisationnelles complexes, et régler également le problème de l'opportunisme lors des échanges inter entreprises. C'est une théorie utile pour appréhender l'évolution des structures organisationnelles modernes et complexes, en expliquant que l'objectif des relations inter organisationnelles se focalise sur la réduction des coûts de transaction.

- L'intégration des Supply Chains selon l'optique de la théorie des coûts de transaction

Lambert et Cooper (2000) affirment que le Supply Chain Management, est l'intégration des processus depuis le dernier utilisateur, en passant par les fournisseurs qui offrent des prestations et de l'information, qui garantissent de la valeur ajoutée pour les clients et pour toutes les parties prenantes. L’intégration des Supply Chains représente le sujet majeur, et le facteur clé de succès du Supply Chain Management. Cette intégration encourage le partage d'information concernant les processus clés ; les producteurs peuvent réagir d'une manière plus flexible aux demandes individuelles des clients, réduire le temps de livraison et baisser le niveau des stocks (Clark et Lee, 2000 ; Barrat, 2004).

Selon l'optique de la théorie des coûts de transaction, l'intégration des Supply Chains peut être vue comme un mode de gouvernance spécial (Das et al, 2006). Sa formation est influencée par les investissements spécifiques réalisés par les partenaires (Bates et Hollingrowth, 2004 ; Heide et John, 1990 ; Rinehart et al, 2004), et sa formation induit également une réduction des coûts et de l'incertitude et améliore la performance ( D'aveni et Ravenscarft, 1994 ; da Silveira et Arkader, 2007 ; Handfield et Regatz, 1999 ; Rosenzweig et al, 2003). L’intégration des Supply Chains réduit également les coûts de transaction, à travers l'établissement des relations à long terme avec les partenaires. L’intégration des Supply Chains réduit les coûts résultant de la conclusion des contrats, et les coûts de négociation, parce que les producteurs partagent les informations avec leurs clients en temps réel, et le temps nécessaire pour la négociation devient restreint.

La réduction des coûts de transaction sont minimisés par l’intégration des Supply Chains, à partir de la construction de la confiance et des relations à long terme avec les partenaires. A travers l'intégration des Supply Chains, l'information est partagée tout au long de la Supply Chain, et elle est disponible en temps réel pour tous les maillons de la Supply Chain. Cela entraîne une grande visibilité et permet d'éviter le retard et la distorsion de l'information et par conséquent, réduire les coûts de transaction engendrés 
par l’opportunisme. En revanche, le facteur clé de la réussite d'une intégration efficace entre les partenaires de la Supply Chain, est la confiance. Sans la fondation de la confiance entre les différents acteurs de la Supply Chain, aucun type d'intégration n’atteindra le succès attendu. Il faut noter que la confiance représente un élément primordial et antécédent à l’intégration des Supply Chains.

\section{La théorie de l'engagement et de la confiance (Robert M. Morgan et Shelby D. Hunt)}

- $\quad$ Présentation générale de la théorie de l’engagement et de la confiance

La théorie de l'engagement et de la confiance fondée par Morgan et Hunt, est une théorie inspirée du domaine du marketing, qui stipule que la réussite des relations en marketing, exige la construction de l'engagement et de la confiance. L'élaboration de la théorie de l'engagement et de la confiance était réalisée après le constat, que l'aspect coopératif des comportements économiques était négligé. Les économistes parlent des théories de la pure compétition, et ignorent les théories de la pure coopération (Alderson, 1965). Toutefois, le plus important facteur de l'efficacité des organisations sociales est la volonté de toute personne à avoir confiance en une autre personne, et de l'efficience des groupes sociaux qui dépend de l'existence ou l'absence de la confiance (Rotter, 1967).

Morgan et Hunt (1994) indiquent que l'engagement et la confiance sont les «clés », parce qu’ils encouragent les marketers à protéger et à investir dans les relations, à travers la coopération avec les partenaires, à résister aux événements imprévus et à bénéficier des relations construites pour le long-terme et, finalement, à minimiser les risques potentiels inhérents aux relations, en ayant confiance en ses partenaires qu'ils ne vont pas agir d'une manière opportuniste. Bref, pour Morgan et Hunt, l’engagement et la confiance engendrent directement les comportements coopératifs, qui participent au succès des relations dans le domaine du marketing.

L’engagement, selon Morgan et Hunt, est une relation pendant laquelle un associé croit que la relation actuelle avec les autres associés est très importante, quant aux efforts qui doivent être fournis pour la maintenir, et s'assure qu'elle va durer indéfiniment. La confiance, est quand une partie croit au sérieux et à l'intégrité d'une autre partie, affirment Morgan et Hunt. Comme l'engagement, la confiance, a été largement étudiée dans la littérature des échanges sociaux (Fox, 1994 ; Scanzani, 1979). Dans les alliances stratégiques, Sharman (1992) montre que le plus grand blocage au succès des alliances est le manque de confiance. Morgan et Hunt théorisent que la confiance est fondamentale dans tous les échanges sociaux, et que la confiance conduit à la coopération et à la collaboration, ainsi que la construction des relations à long terme avec les partenaires. La coopération 
selon les deux auteurs, est le seul résultat qui est directement relatif et influencé par la confiance. Les travaux théoriques ainsi que les recherches empiriques indiquent que la confiance mène à la coopération.

- $\quad$ La confiance et l'intégration des Supply Chains

La littérature existante a amplement indiqué que la confiance est pertinente dans le Supply Chain Management, et a montré que la confiance, est un facteur prédictif des responsables des résultats positifs et de la performance dans le contexte du Supply Chain ( Irland et Webb, 2007 ; Laaksonen et al, 2009 ; Panayides et Venus, 2009 ; Lee et al, 2010).

La corrélation positive qui existe entre la confiance et la performance des Supply Chain, demeure notable dans plusieurs types d'industries, notamment, celle de l'automobile, des fournitures d'ordinateurs et des composantes électroniques (Nooteboom et al, 1997 ; Sako et Helper, 1998 ; Dyer et Chu, 2003 ; Capaldo, 2014). Ainsi, la performance générée par la confiance a été vérifiée par plusieurs chercheurs en termes de réduction des coûts et de développement de la flexibilité (Laaksonen et al, 2009 ; Narasimhan et Nair, 2005). Dans les relations inter organisationnelles, les participants dans une Supply Chain estiment que leurs associés feront ce qui est meilleur pour le système global. En faisant confiance, les organisations sont disposées à collaborer avec leurs partenaires, parce qu'elles présument que ces parties ne se comporterons pas d'une manière opportuniste (Barney et Hansen, 1994 ; Bradach et Eccles, 1989 ; Lai et al, 2012).

En effet, la confiance fonctionne également dans la Supply Chain comme un mécanisme informel de gouvernance, qui facilite la coordination inter organisationnelle ( Bradach et Eccles, 1989 ; Heide, 1994 ; Capaldo, 2014). Finalement, quand la confiance est présente dans la Supply Chain, les partenaires tendent à se comporter d'une manière altruiste, pour le bien de la Supply Chain entière. Le précieux effet de la confiance dans les relations inter organisationnelles est bien traité dans la littérature. Il est également analysé selon plusieurs théories dont la plus notable reste celle de la théorie des coûts de transaction. Dans le cadre de la théorie de cette dernière, la confiance réduit la recherche, le coût de surveillance et de contrôle, aussi bien que les incertitudes dans le partage d'informations (Dyer et Chun, 2003 ; Zaheer et al, 1998).

Dans les études du management stratégique, la confiance a été identifiée comme une cause déterminante des relations inter organisationnelles, associée à l'adaptabilité et au comportement des associés ( Mohr et Spekman, 1994; Yang, 2009), ajoutant également, que la confiance ne laisse pas de place pour l'opportunisme. Les recherches empiriques ont confirmé que la confiance et l'opportunisme sont des substituts l'un de l'autre ( Zaheer et Venkatraman, 1995 ; Dyer et Chun, 2003 ; Cavusgil et al, 2004). 


\section{L'étude de l’impact des déterminants de la culture nationale sur l'intégration relationnelle des Supply Chains : Contexte Marocain}

Dans cet article, nous allons nous focaliser sur l'aspect relationnel de l'intégration des Supply Chains. Du coup, la prise en compte des aspects culturels, éthiques et relationnels lors de l'analyse de l'intégration des Supply Chains demeure fondamentale. Nous remarquons à travers notre revue de la littérature que la majorité des recherches, se focalisent d'une manière quasi-exclusive sur les éléments opérationnels et technologiques, permettant la mise en place d'une intégration efficace des maillons de la Supply Chain. D’où le choix de notre concentration totale sur l'optique relationnelle de l’intégration des Supply Chain.

\section{La culture nationale}

- $\quad$ La distance hiérarchique

Diverses études ont montré le fort degré hiérarchique existant au Maroc (Mezouar et Semeriva, 1998 ; Al Maache, 2002 ; Eddakir, 2003 ; Nouiga, 2003 ; House et al, 2004). Et le projet GLOBE réalisé par House, confirme le plus haut score de la distance hiérarchique du Maroc, sur 62 pays faisant partie de l'étude. Ces résultats rejoignent ceux d'Hofstede (1994), qui montrent que généralement, les pays avec un indice de distance hiérarchique élevé sont des pays, également, collectivistes contrairement aux pays individualistes où le degré de la distance hiérarchique est faible.

- L'orientation individualiste ou communautaire

Beaucoup d’études se sont intéressées au contexte marocain (Hofstede, 1980, 2001 ; Mezouar et Semeriva 1998 ; Al Maache, 2002 ; Eddakir, 2003, Nouiga, 2003 ; House et al, 2004 ; Matsumoto, 2006 ; Hmaida, 2010), et indiquent que la culture marocaine est caractérisée par un niveau élevé de collectivisme. En addition, les résultats d’Hofstede classifient le Maroc parmi les pays collectivistes. Al Maache (2002) dans son étude sur le style de gestion au Maroc, affirme que ce dernier connaît un niveau élevé de collectivisme.

- $\quad$ La relation à l'incertitude et à l'ambigüité

Les études associées au contexte marocain, montrent que la culture marocaine est caractérisée par un degré faible du contrôle de l'incertitude (Nouiga, 2003 ; Eddakir, 2003). Selon Al Maache (2003), les marocains attribuent ce faible niveau de contrôle de l'incertitude et de l'ambiguïté à l'Islam et à la notion du Maktoub. En effet, la notion du Maktoub soulage les personnes des inquiétudes et de l'anxiété causées par les événements imprévisibles.

- $\quad$ L’orientation masculine ou féminine des valeurs

Cette dimension présente un problème dans le contexte marocain. Les travaux qui se sont intéressés à ce dilemme, exposent des résultats 
contradictoires. A côté du score d’Hofstede attribué au Maroc (53 pour les valeurs masculines et 47 pour les valeurs féminines), le travail de Nouiga (2003) et celui de Balambo (2014), montrent que la culture marocaine est dominée par un fort degré de féminité.

- L'orientation à court terme et l'orientation à long terme

Hofstede (2010) montre que dans certaines cultures musulmanes orientées court terme, l'hésitation des migrants à changer de nationalité, qui mène à avoir deux passeports, traduit moins la fierté nationale que la religiosité. Une culture nationale comme celle du Maroc, comme l'indique Hofstede (2010), encourage vivement ça. Elle ne conçoit pas qu'un enfant d'un grand pays puisse renoncer à sa citoyenneté. A travers, le passage cité par Hofstede (Cultures et organisations: Nos programmations mentales, Geert Hofstede, Gert Jan Hofstede, Michael Minkov, 2010), nous pouvons conclure que la culture marocaine représente une culture dont l'orientation est à court terme, basée sur le respect des traditions et de la conformité aux normes sociales.

- $\quad$ La religiosité

La société marocaine, est justement, en grande partie, marquée par la religion (Balambo, 2012,2013). Un éventail de travaux montrent la religiosité de la société marocaine ( Geertz, 1973, d’Iribarne, 1997, Al Maache, 2002, Bourquia et al, 2007, Tory et al, 2007). Finalement, comme l'ont déjà confirmé, d’Iribarne (1997) et Geertz (1973), la culture marocaine est caractérisée par un attachement fort à la religion.

Dans la partie suivante nous allons voir quel type de confiance peut être généré à travers ces déterminants de la culture marocaine pour, en fin, mettre en relation la culture nationale et la confiance.

\section{La confiance : (la confiance cognitive VS la confiance affective)}

A travers notre revue de la littérature sur les types de confiance existant, nous avons adopté la typologie de Mc Allister (1995) ; qui explique à notre sens tous les critères sous-jacents pour prendre la décision de faire confiance. La typologie de Mc Allister englobe, la confiance cognitive et la confiance affective. La confiance cognitive, fondée sur les informations individuelles, sur la fiabilité et la dépendance à l’égard de l'autre. Cette confiance dite rationnelle, se base sur les caractéristiques objectives attribuées aux partenaires (Mc Allister, 1995 ; Tyler et Kramer, 1996), comme la responsabilité, l’intégrité, la crédibilité et la fiabilité. Alors que la confiance affective se base sur l'attention, sur les émotions interpersonnelles entre les individus, ces liens affectifs constituent la base de la prise de décision de faire confiance aux autres. Cette confiance s'appuie sur la bienveillance, l'engagement et le respect mutuel. 


\section{Liens entre la culture nationale et la confiance : Hypothèses}

Comme déjà cité précédemment, la culture marocaine est une culture qui est marquée par un fort degré de distance hiérarchique. Cette distance hiérarchique influence fortement le processus d'attribution de la confiance. En fait, dans les pays à forte distance hiérarchique, l'importance est donnée au statut social et aux signes extérieurs. Par conséquent, le processus d'attribution de la confiance dans ces pays, se fait sur la base des critères affectifs et engendre comme résultat, une confiance affective. Compte tenu de ces conclusions, nous formulons notre première hypothèse : H1 : Une culture à forte distance hiérarchique est positivement associée à une confiance affective.

La culture marocaine, est une culture marquée par les valeurs communautaires, basées sur l'importance de l'entourage et sur la prédisposition à l'entraide (Franceso et Chen, 2004). Les partenaires ont tendance à construire des liens affectifs, et la communauté prend un essor sur l'individu. Cette cohésion perçue entre les partenaires se fonde, en effet, sur les liens affectifs. La personne bénéficie de la bienveillance et de la protection du groupe, qui lui doit loyauté en contrepartie. Le processus de développement de la confiance entre les partenaires se fait alors sur des bases affectives (Williams, 2001 ; Huff et Kelley, 2005 ; Ferrin et al. 2006 ; El Akremi et al. 2007). Ces conclusions confortent l'élaboration de la deuxième hypothèse : H2 : Une culture communautaire est positivement associée à une confiance affective.

D’après notre revue de la littérature, la culture marocaine représente une culture à faible contrôle de l'incertitude. Dans des cultures similaires, la prise de risque est considérée comme un fait habituel. Le comportement des individus n’est pas marqué par une anxiété et une inadaptation aux situations nouvelles. Les personnes sont beaucoup plus ouvertes et tolèrent les différences d'avis, et ne respectent que les règles formelles demeurant fondamentales. Contrairement aux cultures à fort contrôle de l'incertitude. Les individus appartenant à une telle culture apparaissent plus flegmatiques, relâchés et contemplatifs (Hofstede et Bond, 1988). Leurs relations avec l'autre sont marquées par la recherche de bienveillance et d'attachement (Elahee et al. 2002), ils ne sont pas préoccupés par l'ambiguïté de l'avenir et leurs choix se fondent sur l'intuition et l'affectivité. Dans une telle culture, la confiance est développée à travers des processus affectifs (El Akremi et al. 2007). Ces éléments théoriques, déjà cités ci-dessus, nous amènent vers la formulation de la troisième hypothèse : H3 : Une culture à faible contrôle de l'incertitude est positivement associée à une confiance affective

Notre revue de la littérature nous a révélé que la culture marocaine est une culture marquée par une orientation féminine des valeurs. Dans des cultures similaires, les membres sont supposés être modestes, tendres et 
préoccupés par la qualité de la vie (Hofstede, 1994), avec une orientation vers la coopération, la bienveillance et l'altruisme (Hofstede, 1991). Les individus des cultures féminines mettent l'accent sur les relations sociales, l'entraide où l'intérêt collectif domine l'individuel. Ils bâtissent leur confiance sur la base des valeurs citées (Geffen et Heart, 2006). L'appartenance à une culture féminine favorise le développement d'une confiance fondée sur l'affect (Doney et al, 1998 ; El Akremi et al. 2007).

Ces conclusions vont conforter la formulation de la quatrième hypothèse : H4 : Une culture à orientation féminine est positivement associée à une confiance affective. Nous avons conclu à travers notre revue de la littérature, que la culture marocaine fait partie des cultures identifiées par une orientation à court terme, et marquée par le respect des traditions, des obligations sociales, l'altruisme et la création des relations. Nous pouvons conclure que la culture à orientation court terme, construit son processus de prise de décision d'attribuer la confiance, sur des normes relationnelles, affectives et basées sur le respect de l'autre. A travers ces éléments, nous pouvons formuler notre cinquième hypothèse : H5 : Une culture à orientation court terme est positivement associée à une confiance affective.

Finalement, et pour la simple raison de respecter au mieux notre contexte de recherche, nous avons intégré la religiosité comme sixième déterminant caractérisant la culture nationale.

La revue de la littérature nous a indiqué que la culture marocaine est marquée par une forte religiosité. Les individus d'une telle culture, ignorent toute projection dans le futur qui implique une mobilisation de processus objectifs de prédiction, au profit de considérations abstraites et métaphysiques. Les cultures à forte religiosité restent marquées par une forte affectivité dans les relations avec les partenaires, qui se construit autour d'une idéologie, de principes moraux communs et de ressemblances identitaires (Hernandez, 2001). Dans cette culture, le processus de construction de la confiance se base sur un processus affectif (El Akremi et al. 2007).

Ces éléments supportent notre sixième hypothèse : H6 : Une culture à forte religiosité est positivement associée à une confiance affective.

\section{Relation entre la confiance et l'intégration des Supply Chain : Hypothèse}

A travers notre revue de la littérature, nous avons adopté la typologie de l'intégration indiquée dans les travaux de Stank et al. (2001), Zhao et al. (2004) et Baofeng et al. (2004). Cette typologie se compose de deux types d’intégrations; à savoir l'intégration fonctionnelle et l'intégration relationnelle. 
L’intégration fonctionnelle est basée sur des éléments impersonnels, objectifs qui permettent d'intégrer une Supply Chain. Tandis que l'intégration relationnelle, celle qui nous intéresse dans ce travail de recherche, est fondée sur le développement des actions de coordination, de collaboration qui permet de bâtir " une osmose relationnelle et culturelle » (Baratt, 2004). Une osmose qui ne pourrait être réalisée sans un investissement émotionnel dans la relation de confiance, manifestant une attention sincère et particulière au bien-être des autres (McAllister 1995). Un investissement qui donne une attention singulière aux critères relationnels et sociaux de la relation, plutôt que des attributs instrumentaux (Williams, 2001).

Ces caractéristiques correspondent aux déterminants de la confiance affective (McAllister1995). La confiance affective est identique à la prédisposition du partenaire à avoir un comportement ouvert vis à vis de l'autre. Elle est marquée par une volonté de faire plus que ce qui est formellement prévu (Sako 1992). Dans les travaux récents de Ha et al.(2011), la confiance affective est identifiée, comme ayant un impact significatif sur l'intégration relationnelle dans des Supply Chains, notamment sur la volonté de partage de l'information et de partage des risques et des gains.

Les éléments que nous venons de citer, nous permettent de formuler notre septième et dernière hypothèse : H7: Une confiance affective est positivement associée à l'intégration relationnelle des Supply Chains.

\section{Conclusion}

L'inscription des entreprises marocaines dans les Supply Chains, exige un changement dans les outils et les méthodes de gestion et de partage de l'information, par l'implantation de nouveaux systèmes d'information, qui peut faire l'objet de plusieurs feed-back positifs ou négatifs de la part des responsables et des employés. L'acceptation ou le refus est inhérent à un certain nombre de paramètres relatifs à la culture nationale. Comme l'explique Philippe d'Iribarne, la culture est un système de sens, à travers lequel l'individu perçoit et interprète une situation ou une action concrète. Ce système de sens, qu'il a constitué au cours de son histoire, est partagé avec les autres membres de sa communauté.

En effet, la culture nationale demeure un élément critique et crucial, puisque son influence est notable sur les valeurs et les modes de vie ( Sun, Horn et Merritt, 2004), sur la perception de la qualité du service et la satisfaction ( Ultshy et al, 2004), sur les négociations ( Adair et Brett, 2005), sur la manière de rechercher l'information ( Doran , 2002), sur les pratiques en matière de publicité ( Taylor et Okazaki, 2006), et sur les comportements et l'éthique ( Elahee, Kirby et Nasif, 2002). Ainsi, nous pouvons estimer que 
la culture nationale, peut avoir des influences sur l'intégration relationnelle des Supply Chains et sur la construction de la confiance entre les partenaires.

Dans le présent papier, nous nous intéressons au positionnement de la culture nationale dans l'intégration des Supply Chains via l'analyse des déterminants de ce concept fondamental, en nous focalisant sur l'aspect relationnel de l'intégration des Supply Chains, autrement dit l'intégration relationnelle des Supply Chains. Ceci, parce que cette intégration relationnelle ou comportementale (Bowersox et al, 1996, 1999, 2000 ; Mentzer et al, 2001) constitue la pierre angulaire du Supply Chain Management (Mentzer et al, 2001).

Pour conclure et compte tenu des conclusions de notre travail, nous rappelons les sept hypothèses que nous avons développées à savoir :

H1 : Une culture à forte distance hiérarchique est positivement associée à une confiance affective ;

H2 : Une culture communautaire est positivement associée à une confiance affective ;

H3 : Une culture à faible contrôle de l'incertitude est positivement associée à une confiance affective ;

H4 : Une culture à orientation féminine est positivement associée à une confiance affective ;

H5 : Une culture à orientation à court terme est positivement associée à une confiance affective ;

H6 : Une culture à forte religiosité est positivement associée à une confiance affective ;

H7 : Une confiance affective est positivement associée à l'intégration relationnelle des Supply Chains.

\section{References:}

Ali A. J., Wahabi R. (1995), Managerial value systems in Morocco, International Studies of Management \& Organization, vol. 25, n³, p. 87-96. Balambo M. A. (2013), Culture nationale et nature de l'intégration des supply chains amont: le cas des équipementiers automobiles marocains, Logistique \& Management, vol. 21, n 4, p. 71-85.

Balambo M. A. (2014), Hofstede's model revisited: an application for measuring the Moroccan national culture, International Journal of Business Quantitative Economics and Applied Management Research, Vol.1, ${ }^{\circ} 3$.

Baofeng Huo, Xiande Zhao, Jeff Hoi Yang Yeung, Power (2005), Relationship Commitment and Supply Chain Integration Between Manufacturer and Supplier, Proceeding of the fifth International Conference on Electronic Business, Hong Kong, December 5-9, p.868-881. 
Barratt M. (2004), Understanding the meaning of collaboration in the supply chain. Supply Chain Management: an international journal, vol.9, n¹, p.3042.

Doney P. M., Cannon J. P., Mullen M. R. (1998), Understanding the influence of national culture on the development of trust, Academy of management review, Vol.23, $n^{\circ} 3$, p.601-620.

El Akremi, A., Ikram Nasr, M., \& Sassi, N. (2007), Impact de la culture nationale sur la confiance interpersonnelle en milieu du travail: analyse comparative entre la France et la Tunisie, Les actes des congrès AGRH.

Haider I. (2014), Collaboration in Supply Chain Management: What it is and How to use it? (An intenational review), International Journal of Research, Vol.1, n6, p.499-507.

Hofstede G. (1993), Cultural constraints in management theories, The Academy of Management Executive, vol.7, n ${ }^{\circ}$, P.81-94.

Hofstede G. (2011), Dimensionalizing cultures: The Hofstede model in context, Online readings in psychology and culture, vol.2, $n^{\circ} 1$, P.8.

Hofstede G. (1983), The cultural relativity of organizational practices and theories, Journal of international business studies, Vol.14, n², P.75-89.

Hofstede G. (1998), Think locally, Act globally: Cultural Constraints in Personnel Management, Management International Review, Vol.38, Cross Cultural and Comparative International Human Ressource Management, p.726.

Mentzer J. T., DeWitt W., Keebler J. S., Min S., Nix N. W., Smith C. D., Zacharia Z. G. (2001). Defining supply chain management, Journal of Business Logistics, Vol.22, n², p.1-25.

Power, D. (2005), Supply chain management integration and implementation: a literature review, Supply chain management: an International journal, vol.10, ${ }^{\circ} 4$, P. 252-263.

Schwartz S. H., Huismans S. (1995), Value priorities and religiosity in four western religions, Social Psychology Quterly, vol.58, n², p.88-107.

Stank T. P., Keller S. B., Closs D. J. (2001), Performance benefits of supply chain logistical integration, Transportation Journal, vol.41, n²/3, p. 32-46. Yvonne M., Everdingen V., Waarts E. (2003), The effect of National Culture on the adoption of innovations, Marketing Letters, Vol.14, n³, p. 217-232. 